\title{
SECTIONS OF SIMPLICES
}

\author{
NAGABHUSHANA PRABHU
}

(Received 28 August 1995 and in revised form 10 October 1995)

ABSTRACT. We show that for $\lfloor d / 2\rfloor \leq k \leq d$, the relative interior of every $k$-face of a $d$-simplex $\Delta^{d}$ can be intersected by a $2(d-k)$-dimensional affine flat. Bezdek, Bisztriczky, and Connelly's results [2] show that the condition $k \geq\lfloor d / 2\rfloor$ above cannot be dropped and hence raise the question of determining, for all $0 \leq k, j<d$, an upper bound on the function $c(j, k ; d)$, defined as the smallest number of $j$-flats, $j<d$, needed to intersect the relative interiors of all the $k$-faces of $\Delta^{d}$. Using probabilistic arguments, we show that

$$
C(j, k ; d) \leq \frac{\left(\begin{array}{l}
d+1 \\
k+1
\end{array}\right)}{\left(\begin{array}{l}
w+1 \\
k+1
\end{array}\right)} \log \left(\begin{array}{l}
d+1 \\
k+1
\end{array}\right), \quad \text { where } w=\min \left(\max \left(\left\lfloor\frac{j}{2}\right\rfloor+k, j\right), d\right) .
$$

Finally, we consider the function $M(j, k ; d)$, defined as the largest number of $k$-faces of $\Delta^{d}$ whose relative interiors can be intersected by a $j$-flat. We show that, for large $d$ and for all $k$ such that $k+j \geq d, M(j, k ; d) \leq f_{\lceil 3 j / 4\rceil-1}(d+1, j)$, where $f_{m}(n, q)$ is the number of $m$-faces in a cyclic $q$-polytope with $n$-vertices. Our results suggest a conjecture about face-lattices of polytopes that if proved, would play a useful role in further studies on sections of polytopes.

Keywords and phrases. Simplex, polytope, face-lattice.

1991 Mathematics Subject Classification. 05B35, 52B05, 52B11, 52B30, 52C07.

1. Introduction. A class of problems of continuing interest concerns studying the intersection of an affine flat with the boundary complex of a $d$-polytope (hereafter called a section of the $d$-polytope). A diverse body of results about this class of problems has been reported in the literature. O'Neil studied the popular problem of determining the maximum number of edges of a $d$-cube that a hyperplane of $R^{d}$ can cut and proved a lower bound of $(d-\lfloor d / 2\rfloor)\left(\begin{array}{c}d \\ \lfloor d / 2\rfloor\end{array}\right)$ [7] (see [3] for follow-up work on this problem). Khovanskii [5] showed that if a hyperplane $H$ intersects a $d$-polytope $P$ (and $H^{+}$and $H^{-}$are the two closed halfspaces of $H$ ), then there exists two faces of $P$, $F_{1} \in H^{+}$and $F_{2} \in H^{-}$such that $\operatorname{dim}\left(F_{1}\right)+\operatorname{dim}\left(F_{2}\right) \geq d-1$. Bezdek et al. showed that a hyperplane can intersect the relative interiors of all the $k$-faces of $d$-polytope only if $\lfloor d / 2\rfloor \leq k$ [2]. For other results, see $[1,4,6,8]$.

The most important problems in the above class concern, however, the properties of sections of $d$-simplices. The importance of sections of simplices stems from the fact that any polytope can be realized as a section of a simplex of suitable dimension. Since a section of a section of a simplex is just another section of the simplex, sections of arbitrary polytopes are (in a certain sense) subsumed under the study of sections of simplices. We also note that linear Programs in standard form (or Karmarkar's form) 
are represented as sections of standard simplices. Yet, surprisingly, little is known about the geometric and combinatorial properties of sections of simplices.

In this paper, we study sections of simplices. Specifically, we show that the relative interiors of all the $k$-dimensional faces of a $d$-dimensional simplex, $k \geq\lfloor d / 2\rfloor$, can be intersected by an affine flat of dimension $2(d-k)$ (hereafter, whenever we talk of a flat intersecting a face, we mean that the flat intersects the relative interior of the face even when the term relative interior is dropped). The result yields a counter-intuitive fact that the relative interiors of all the facets of any $d$-simplex can be intersected by a 2 dimensional plane. Bezdek, Bistriczky, and Connelly's result (mentioned above) shows that the restriction $k \geq\lfloor d / 2\rfloor$ cannot be dropped from our result. In light of Bezdek, Bisztriczky, and Connelly's lower bound and our result, an important problem is to determine, for all $1 \leq j, k<d$, the function $C(j, k ; d)$, defined as the smallest number of $j$-flats needed to intersect the relative interiors of all the $k$-faces of a $d$-simplex. Using probabilistic arguments, we prove the following fairly nontrivial upper bound on $C(j, k ; d)$. Let

$$
w=\min \left(\max \left(\left\lfloor\frac{j}{2}\right\rfloor+k, j\right), d\right)
$$

Then for $1 \leq k, j<d$,

$$
C(j, k ; d) \leq \frac{\left(\begin{array}{l}
d+1 \\
k+1
\end{array}\right)}{\left(\begin{array}{l}
w+1 \\
k+1
\end{array}\right)} \log \left(\begin{array}{l}
d+1 \\
k+1
\end{array}\right) .
$$

Another important problem is to determine, for all $1 \leq k, j<d$, the function $M(j, k ; d)$, defined as the largest number of $k$-faces of a $d$-simplex whose relative interiors can be intersected by a $j$-flat. Using the above definition of $w$, one easily obtains the lower bound

$$
\left(\begin{array}{c}
w+1 \\
k+1
\end{array}\right) \leq M(j, k ; d)
$$

on $M(j, k ; d)$. More interestingly, though, for large $d\left(d>d_{0}\right)$ and $k+j \geq d$, we show that the following is a tight upper bound on $M(j, k ; d)$.

$$
M(j, k ; d) \leq f_{\lceil 3 j / 4\rceil-1}(d+1, j),
$$

where $f_{m}(n, q)$ is the number of $m$-faces in a cyclic $q$-polytope with $n$-vertices. A tight upper bound for $M(j, k ; d)$ when $k+j<d$ is less tractable. We discuss the source of difficulty when $k+j<d$ and conclude the paper with some observations about this case and a conjecture about face lattices of polytopes, that if true, would play a useful role in the study of sections of polytopes.

2. Cutting faces of a fixed dimension. For a discussion of the properties of a cyclic polytope used in the proof of the following theorem, the reader is referred to [4].

THEOREM 1. For $\lfloor d / 2\rfloor \leq k \leq d$, the relative interiors of every $k$-face of a $d$-simplex can be intersected by a $2(d-k)$-dimensional affine flat. 
Proof. Let $T^{d}$ be the standard $d$-simplex,

$$
\Delta^{d}=\left\{\left(Y_{1}, \ldots, Y_{d+1}\right) \in R^{d+1} \mid \sum_{i=1}^{d+1} Y_{i}=1, Y_{i} \geq 0, i=1, \ldots, d+1\right\}
$$

and $P \subset R^{n}$ any $n$-dimensional polytope with $d+1$ facets $(n<d+1)$ that is represented as

$$
P=\left\{X \in R^{n} \mid\left\langle X, N_{i}\right\rangle \leq 1 ; i=1, \ldots, d+1\right\},
$$

where $N_{i}$ 's are the normals to the facets and $\langle$,$\rangle denotes dot product.$

A classic theorem on sections [8, Thm. 12, p. 58] states that there exists an $n$ dimensional affine flat $L \subset R^{d+1}$ such that $P$ is projectively and hence combinatorially equivalent to the polytope $L \cap T^{d}$. In fact, $L$ is the image of $R^{n}$ under the following $\operatorname{map} T_{p}: R^{n} \rightarrow R^{d+1}$,

$$
T_{p}(X)=\frac{\left(\left(1-\left\langle N_{1}, X\right\rangle\right), \ldots,\left(1-\left\langle N_{d+1}, X\right\rangle\right)\right)}{(d+1)-\left\langle\sum_{i=1}^{d+1} N_{i}, X\right\rangle}=\frac{\left(\begin{array}{cc}
1 & -N_{1} \\
\vdots & \vdots \\
1 & -N_{d+1}
\end{array}\right)\left(\begin{array}{c}
1 \\
X_{1} \\
\vdots \\
X_{n}
\end{array}\right)}{(d+1)-\left\langle\sum_{i=1}^{d+1} N_{i}, X\right\rangle},
$$

where $N_{i}$ 's are treated as row vectors.

Now, take $P$ to be a $2 m$-polytope combinatorially isomorphic to a dual-cyclic $2 m$ polytope with $d+1$ facets. A cyclic $2 m$-polytope is $m$-neighborly, i.e., every subset of $m$ vertices of a cyclic $2 m$-polytope forms a face. Therefore, in the dual-cyclic $2 m$ polytope, every subset of $m$ facets intersects in a face. Further, in a dual-cyclic $2 m$ polytope, the intersection of every $k$ facets, $1 \leq k \leq m$, is a $(2 m-k)$-face. Thus, every subset of $j$ facets of the dual-cyclic $2 m$-polytope $P, 1 \leq j \leq m$, intersects in a $(2 m-j)$-dimensional face of $P$. Since $P$ is assumed to have $d+1$ facets, the number of $(2 m-j)$-faces of $P, 1 \leq j \leq m$, is $\left(\begin{array}{c}d+1 \\ j\end{array}\right)$.

Observe that $T_{P}$ maps the relative interior of every ( $2 m-j$ )-face of $P$ into the relative interior of a $(d-j)$-face of $\Delta^{d}$. If $F$ is a $(2 m-j)$-face of $P$ that is the intersection of facets with normals $N_{1}, \ldots, N_{j}$ say, then in $T_{P}(F), Y_{1}=\cdots=Y_{j}=0$. Hence, $T_{P}(F)$ is contained in the $(d-j)$-face of $\Delta^{d}$ that is the intersection of the facets $Y_{1}=0, \ldots, Y_{j}=$ 0 . The foregoing argument also shows that the relative interiors of any two distinct $(2 m-j)$-faces of $P$ are mapped into the relative interiors of two distinct $(d-j)$-faces of $\Delta^{d}$.

Since the number of $(2 m-j)$-faces of $P$ equals the number of $(d-j)$-faces of $\Delta^{d}$ for $1 \leq j \leq m$, we conclude that the $2 m$-flat $L=T_{P}\left(R^{2 m}\right)$ intersects the relative interiors of all the $(d-j)$-faces of $\Delta^{d}$ for $1 \leq j \leq m$.

Now, if we set $m=d-k$, then $1 \leq j \leq m$ implies that $d-j \geq k$. Hence, the $2(d-k)$ flat $L$ intersects the relative interiors of all the faces of dimension $k$ and higher in $\Delta^{d}$, which proves the theorem for the standard simplex $\Delta^{d}$.

But any $d$-simplex is an affine image of the standard $d$-simplex $\Delta^{d}$. Further, affine transformations map affine flats to affine flats and preserve incidence relations. Thus, the theorem is true for any $d$-simplex. 
As mentioned earlier, for $k \leq\lfloor d / 2\rfloor$, it follows from Bezdek, Bisztriczky, and Connelly's result [2] that no proper affine subspace of $R^{d}$ can intersect the relative interiors of all the $k$-faces of a $d$-simplex. Therefore, an important problem is to determine for all $0 \leq k, j<d$, the function $C(j, k ; d)$, defined as the smallest number of $j$-flats, $j<d$, needed to intersect the relative interiors of all the $k$-faces of $\Delta^{d}$. Using probabilistic arguments, we prove

THEOREM 2.

where

$$
C(j, k ; d) \leq \frac{\left(\begin{array}{l}
d+1 \\
k+1
\end{array}\right)}{\left(\begin{array}{l}
w+1 \\
k+1
\end{array}\right)} \log \left(\begin{array}{l}
d+1 \\
k+1
\end{array}\right)
$$

$$
w=\min \left(\max \left(\left\lfloor\frac{j}{2}\right\rfloor+k, j\right), d\right) .
$$

Proof. From Theorem 1, we see that for $w$ as defined above, the relative interiors of all the $k$-faces contained in a $w$-face of $\Delta^{d}$ can be intersected by a $j$-flat. Since every $k$-face in any $w$-face of $\Delta^{d}$ can be intersected by one $j$-flat, an upper bound on the number of $w$-faces needed to cover all the $k$-faces of $\Delta^{d}$ yields an upper bound on the number of $j$-flats needed to intersect all the $k$-faces of $\Delta^{d}$. In the following, we show that $\left(\left(\begin{array}{c}d+1 \\ k+1\end{array}\right) /\left(\begin{array}{c}w+1 \\ k+1\end{array}\right)\right) \log \left(\begin{array}{c}d+1 \\ k+1\end{array}\right) w$-faces suffice to cover all the $k$-faces of $\Delta^{d}$ thereby proving the theorem.

Let $V=\left\{v_{0}, \ldots, v_{d}\right\}$ be the vertex-set of $\Delta^{d}$. Every subset of $w+1$ of the $d+1$ vertices of $V$ determines a $w$-face $W$ which contains $\left(\begin{array}{c}w+1 \\ k+1\end{array}\right) k$-faces of $\Delta^{d}$. Let $W=\left\{W_{1}, \ldots, W_{r}\right\}$ be an $r$-collection of $w$-faces of $\Delta^{d}$, where $W_{i}, 1 \leq i \leq r$, are chosen randomly and independently from among the $\left(\begin{array}{c}d+1 \\ w+1\end{array}\right) w$-faces of $\Delta^{d} ; W_{1}, \ldots, W_{r}$ need not all be distinct.

Consider an arbitrary $k$-face of $K$ of $\Delta^{d}$. Let $\tilde{W}$ be an arbitrary $w$-face of $\Delta^{d}$ ( $\tilde{W}$ need not necessarily belong to $\mathcal{W})$. Then the probability that the $w$-face $\tilde{W}$ contains the $k$-face $K$ is

$$
\begin{aligned}
p[k \subset \tilde{W}] & =\frac{\left(\begin{array}{c}
d-k \\
w-k
\end{array}\right)}{\left(\begin{array}{l}
d+1 \\
w+1
\end{array}\right)}=\frac{(d-k) !(d-w) !(w+1) !}{(w-k) !(d-w) !(d+1) !} \\
& =\frac{(d-k) !(w+1) !(k+1) !}{(w-k) !(d+1) !(k+1) !}=\frac{\left(\begin{array}{c}
w+1 \\
k+1
\end{array}\right)}{\left(\begin{array}{l}
d+1 \\
k+1
\end{array}\right)} .
\end{aligned}
$$

Therefore,

$$
P[K \not \subset \tilde{W}]=1-\frac{\left(\begin{array}{l}
w+1 \\
k+1
\end{array}\right)}{\left(\begin{array}{l}
d+1 \\
k+1
\end{array}\right)} .
$$

The probability that none of the randomly chosen $w$-faces of $\mathcal{W}$, namely, $W_{1}, \ldots, W_{r}$, contains $K$ is hence 


$$
P\left[\left(K \not \subset W_{1}\right) \wedge \cdots \wedge\left(K \not \subset W_{r}\right)\right]=\left(1-\frac{\left(\begin{array}{c}
w+1 \\
k+1
\end{array}\right)}{\left(\begin{array}{l}
d+1 \\
k+1
\end{array}\right)}\right)^{r}
$$

Label the $k$-faces of $\Delta^{d}, K_{1}, \ldots, K_{\left(\begin{array}{c}d+1 \\ k+1\end{array}\right)}$. Let $A_{i}$ be the event that $K_{i}$ does not belong to any of the $w$-faces of $\mathcal{W}, 1 \leq i \leq\left(\begin{array}{l}d+1 \\ k+1\end{array}\right)$. Then the probability that a randomly chosen $r$-collection of $w$ - faces $\mathcal{W}$ does not contain at least one $k$-face is

$$
P\left[A_{1} \vee \cdots \vee A_{\left(\begin{array}{c}
d+1 \\
k+1
\end{array}\right)}\right] \leq\left(\begin{array}{l}
d+1 \\
k+1
\end{array}\right)\left(1-\frac{\left(\begin{array}{c}
w+1 \\
k+1
\end{array}\right)}{\left(\begin{array}{l}
d+1 \\
k+1
\end{array}\right)}\right)^{r}
$$

Therefore, if $P\left[A_{1} \vee \cdots \vee A_{\left(\begin{array}{l}d+1 \\ k+1\end{array}\right)}\right]<1$, then $P\left[\bar{A}_{1} \wedge \cdots \wedge \bar{A}_{\left(\begin{array}{c}d+1 \\ k+1\end{array}\right)}\right]>0$ and hence some $r$-collection of $w$-faces must cover all the $k$-faces of $\Delta^{d} . \bar{A}_{i}$ denotes the complement of the event $A_{i}$. Thus, we want

$$
\left(\begin{array}{l}
d+1 \\
k+1
\end{array}\right)\left(1-\frac{\left(\begin{array}{l}
w+1 \\
k+1
\end{array}\right)}{\left(\begin{array}{l}
d+1 \\
k+1
\end{array}\right)}\right)^{r}<1 \quad \text { or } \quad r \ln \left(1-\frac{\left(\begin{array}{l}
w+1 \\
k+1
\end{array}\right)}{\left(\begin{array}{l}
d+1 \\
k+1
\end{array}\right)}\right)+\ln \left(\begin{array}{l}
d+1 \\
k+1
\end{array}\right)<0
$$

or

$$
r>\frac{-\ln \left(\begin{array}{l}
d+1 \\
k+1
\end{array}\right)}{\ln \left(1-\left(\begin{array}{c}
w+1 \\
k+1
\end{array}\right) /\left(\begin{array}{l}
d+1 \\
k+1
\end{array}\right)\right)}
$$

The direction of the inequality is changed in equation (15) since, for $w<d,\left(\begin{array}{c}w+1 \\ k+1\end{array}\right)<$ $\left(\begin{array}{c}d+1 \\ k+1\end{array}\right)$ and hence

$$
\ln \left(1-\frac{\left(\begin{array}{c}
w+1 \\
k+1
\end{array}\right)}{\left(\begin{array}{l}
d+1 \\
k+1
\end{array}\right)}\right)<0
$$

For $0<x<1,-\ln (1-x) \geq x$ since $f(x)=e^{-x}-1+x \geq 0$ for $0<x<1(f(0)=0$ and $f^{\prime}(x)>0$ for $\left.0<x<1\right)$. Hence,

$$
r>\frac{\ln \left(\begin{array}{l}
d+1 \\
k+1
\end{array}\right)}{\left(\begin{array}{c}
w+1 \\
k+1
\end{array}\right) /\left(\begin{array}{l}
d+1 \\
k+1
\end{array}\right)} \Rightarrow r>\frac{-\ln \left(\begin{array}{l}
d+1 \\
k+1
\end{array}\right)}{\ln \left(1-\left(\begin{array}{c}
w+1 \\
k+1
\end{array}\right) /\left(\begin{array}{l}
d+1 \\
k+1
\end{array}\right)\right)}
$$


Therefore, for any $r>\left(\left(\begin{array}{c}d+1 \\ k+1\end{array}\right) /\left(\begin{array}{c}w+1 \\ k+1\end{array}\right)\right) \ln \left(\begin{array}{c}d+1 \\ k+1\end{array}\right), P\left[\bar{A}_{1} \wedge \cdots \wedge \bar{A}_{\left(\begin{array}{c}d+1 \\ k+1\end{array}\right)}\right]>0$ and hence there exists a collection of $\left(\left(\begin{array}{c}d+1 \\ k+1\end{array}\right) /\left(\begin{array}{c}w+1 \\ k+1\end{array}\right)\right) \ln \left(\begin{array}{c}d+1 \\ k+1\end{array}\right) w$-faces of $\Delta^{d}$ which covers all the $k$-faces of $\Delta^{d}$ whose relative interiors can be intersected by a $j$-flat.

From the discussion at the beginning of the proof of Theorem 2, we obtain the lower bound

$$
\left(\begin{array}{c}
w+1 \\
k+1
\end{array}\right) \leq M(j, k ; d)
$$

(see the statement of Theorem 2 for the definition of $w$ ). In the following, we prove a nontrivial upper bound on $M(j, k ; d)$. For $k+j \geq d$ (and only when $k+j \geq d$ ), we can assume, without loss of generality, that any $j$-flat J intersecting the $k$-faces of $\Delta^{d}$ actually intersects the $k$-faces in general position. That is

LEMMA 1. For every $j$-flat J, $1 \leq j<d$, there exists another $j$-flat $\tilde{\mathrm{J}}$ (obtained by perturbing $\mathrm{J})$ such that for $k \geq(d-j)$,

(1) $\tilde{J}$ intersects the relative interiors of a $k$-face of $\Delta^{d}$ if and only if $\mathrm{J}$ does.

(2) If $\tilde{\mathrm{J}}$ intersects the relative interiors of a $k$-face $K$, then $\operatorname{dim}(\tilde{\mathrm{J}} \cap K)=k+j-d$.

Proof. The $j$-flat $\mathrm{J}$ can be represented as the solution set of a system of $d-j$ equations in $d$ variables, i.e.,

$$
j=\left\{x \in R^{d} \mid A x=b ; A:(d-j) \times d \text { matrix; } \operatorname{rank}(A=d-j)\right\} .
$$

Let $\left\{K_{1}, \ldots, K_{r}\right\}$ be the set of all the $k$-faces whose relative interiors are intersected by J. Let $S_{i}$ be the affine span of $K_{i}, 1 \leq i \leq r$. Then $S_{i}$ can be represented as a system of $d-k$ linear equation in $d$ variables. Let

$$
S_{i}=\left\{x \in R^{d} \mid C_{i} x=\alpha_{i}\right\},
$$

where $C_{i}$ is a $(d-k) \times d$ matrix and $\operatorname{rank}\left(C_{i}\right)=d-k$.

Since $J \cap S_{i}=\left\{x \in R^{d} \mid A x=b ; C_{i} x=\alpha_{i}\right\}, \operatorname{dim}\left(J \cap S_{i}\right)=d-\operatorname{rank}\left[\left(\begin{array}{c}A \\ C_{i}\end{array}\right)\right]$. Hence, $\operatorname{dim}\left(J \cap S_{i}\right)=\operatorname{dim}\left(J \cap K_{i}\right)=k+j-d$ if and only if the rank of the matrix $M_{i}=\left(\begin{array}{c}A \\ C_{i}\end{array}\right)=$ $(d-k)+(d-j)=$ the number of rows in $M_{i}$.

Clearly, by an infinitesimal perturbation $\delta A$, one can ensure that $\tilde{M}=\left(\begin{array}{c}A^{+} \delta A \\ C_{i}\end{array}\right)$ has $\operatorname{rank}(d-j)+(d-k)$ for all $1 \leq i \leq r$. Further, since the solution set J depends continuously on the entries in $A$, if the absolute value of each element of $\delta A$ is bounded by a sufficiently small $\epsilon>0$ and

$$
\tilde{\mathrm{J}}=\left\{x \in R^{d} \mid(A+\delta A) x=b\right\},
$$

then $\tilde{\mathrm{J}} \cap$ relative interior $\left(K_{i}\right) \neq \varnothing$ for $1 \leq i \leq r$.

The restriction $k+j-d \geq 0$ in the statement of the lemma cannot be done away with because if $k+j<d$, then the $(2 d-k-j) \times d$ matrix $M_{i}$ (see above) would have more rows than columns. Therefore, in the system of equations

$$
A x=b, \quad C_{i} x=\alpha_{i},
$$


if the rows of $A$ are perturbed to include full row-rank in the matrix $M$, the resulting system could become inconsistent. In order to make an inconsistent system consistent, the right-hand side constants need to be perturbed and the required perturbations of the right-hand side constants may not be small enough to ensure that $\tilde{J}$ will continue to intersect the relative interior of every $k$-face that is intersected by J. The restriction $k+j-d \geq 0$ in Lemma 1 carries over to the following Theorem 3. The problem of proving Theorem 3 when $k+j<d$ is harder and we present some observations about the following Theorem 3.

THEOREM 3. For large $d$ and for all $k$ such that $k+j \geq d$,

$$
M(j, k ; d) \leq f_{\lceil 3 j / 4\rceil}(d+1, j) .
$$

Proof. To determine $M(j, k ; d)$, it is sufficient to restrict our attention to the $j$ flats that intersect the interior of $\Delta^{d}$ (if the $j$-flat does not intersect the interior of $\Delta^{d}$, then it intersects the relative interiors of only the $k$-faces that are contained in a proper subface of $\Delta^{d}$. However, clearly, $M\left(j, k ; d_{1}\right) \leq M\left(j, k ; d_{2}\right)$ if $\left.d_{1} \leq d_{2}\right)$. Let $\mathrm{J}$ be a $j$-flat intersecting the interior of $\Delta^{d}$. Then

$$
P=J \cap \Delta^{d}
$$

is a $j$-polytope.

If the $j$-flat $\mathrm{J}$ intersects the relative interior of a $k$-face $\mathrm{K}$ of $\Delta^{d}$, then from Lemma 1 , we may assume, without loss of generality, that $\operatorname{dim}(K \cap J)=k+j-d$. Therefore, the number of $k$-faces of $\Delta^{d}$ whose relative interiors are intersected by the $j$-flat $\mathrm{J}$ is at most the number of $(k+j-d)$-faces in P. Now, P has at most $d+1$ facets as the following argument shows. If $F$ is any facet of $\Delta^{d}$ and $H$ the hyperplane determined by $F$, then $H \cap P$ is a face of $\mathrm{P}$ (possibly empty). Hence, $H$ can contain at most one facet of P. Repeating this argument for the $d+1$ facets of $\Delta^{d}$, the claim follows. Therefore, we conclude that $M(j, k ; d)$ is at most the number of $(k+j-d)$-faces in a $j$-polytope with $d+1$ facets. Dually, $M(j, k ; d)$ is at most the number of $(d-k-1)$-faces in a $j$-polytope with $d+1$ vertices. By the Upper Bound Theorem, we conclude that

$$
M(j, k ; d) \leq f_{d-k-1}(d+1, j),
$$

where $f_{d-k-1}(d+1, j)$ is the number of $(d-k-1)$-faces in a cyclic $j$-polytope with $d+1$ vertices. In order to determine an upper bound valid for all $k$ (i.e., independent of $\mathrm{k}$ ), we need to maximize the function $f_{d-k-1}(d+1, j)$ with respect to $k$. Unfortunately, even though explicit formulae are known for $f_{m}(n, j)$ for all values of $m, n$, and $j$, the problem of maximizing $f_{d-k-1}(d+1, j)$ with respect to $k$ is not tractable. However, in the large $d$ limit, we can determine the maximum of $f_{d-k-1}(d+1, j)$ with respect to $k$ as follows.

We have

$$
\begin{aligned}
f_{t}(n, 2 m) & =\sum_{r=1}^{m} \frac{n}{n-4}\left(\begin{array}{c}
n-r \\
r
\end{array}\right)\left(\begin{array}{c}
r \\
t+1-r
\end{array}\right) \\
& =\sum_{r=1}^{m} \frac{n(n-r-1)(n-r-2) \cdots(n-2 r+1)}{r !}\left(\begin{array}{c}
r \\
t+1-r
\end{array}\right), \quad 0 \leq t \leq 2 m-1,
\end{aligned}
$$




$$
\begin{aligned}
f_{t}(n, 2 m+1) & =\sum_{r=0}^{m} \frac{t+2}{n-4}\left(\begin{array}{c}
n-r \\
r+1
\end{array}\right)\left(\begin{array}{c}
r+1 \\
t+1-r
\end{array}\right) \\
& =\sum_{r=0}^{m} \frac{(t+2)(n-r-1)(n-r-2) \cdots(n-2 r)}{(r+1) !}\left(\begin{array}{c}
r+1 \\
t+1-r
\end{array}\right), \quad 0 \leq t \leq 2 m .
\end{aligned}
$$

Both $f_{t}(n, 2 m)$ and $f_{t}(n, 2 m+1)$ can be viewed as polynomials of $n$ whose coefficients are functions of $t$ and $m$. In order to determine the $t$ at which $f_{t}(n, 2 m)$ and $f_{t}(n, 2 m+$ 1 ) are maximized for large $n$, we need to maximize the coefficient of the leading term in the polynomials with respect to $t$.

The coefficient of the leading term in $f_{t}(n, 2 m)$ is

$$
\frac{1}{m !}\left(\begin{array}{c}
m \\
t+1-m
\end{array}\right)
$$

which is maximized at $t+1-m=\lceil m / 2\rceil$ or at $t=m-1+\lceil m / 2\rceil$. On the other hand, the coefficient of the leading term in $f_{t}(n, 2 m+1)$ is

$$
\frac{(t+2)}{(m+1) !}\left(\begin{array}{c}
m+1 \\
t+1-m
\end{array}\right)=\frac{(t+2)}{(m+1) !}\left(\begin{array}{c}
m+1 \\
(t+2)-(m+1)
\end{array}\right)
$$

which is maximized at $(t+2)-(m+1)=\lceil(m+1) / 2\rceil$ or $t=m-1+\lceil(m+1) / 2\rceil$ (it is easy to verify that $x\left(\begin{array}{c}y \\ x-y\end{array}\right)$ is maximized at $\left.x=y-\lceil y / 2\rceil\right)$. To summarize, if for large $n, f_{t_{\max }}(n, s) \geq f_{i}(n, s), 0 \leq i \leq s-1$, then

$$
t_{\max }= \begin{cases}m-1+\left\lceil\frac{m}{2}\right\rceil, & \text { if } s=2 m, \\ m-1+\left\lceil\frac{m+1}{2}\right\rceil, & \text { if } s=2 m+1 .\end{cases}
$$

Table 1 expresses $t_{\max }$ in terms of the dimension $s$.

TABLE 1.

\begin{tabular}{|c|c|c|}
\hline$s=2 m$ & $s=2 m+1$ & \multirow{2}{*}{$m$} \\
\cline { 1 - 2 }$t_{\max }=m-1+\left\lfloor\frac{m}{2}\right\rfloor$ & $t_{\max }=m-1+\left\lfloor\frac{m+1}{2}\right\rfloor$ & \\
\hline$s=4 l$ & $s=4 l+1$ & \\
\cline { 1 - 2 }$t_{\max }=\left\lceil\frac{3 s}{4}\right\rceil-1$ & $t_{\max }=\left\lceil\frac{3 s}{4}\right\rceil-1$ & \\
\cline { 1 - 2 }$=4 l+2$ & $s=4 l+1$ & \\
\cline { 1 - 2 }$t_{\max }=\left\lceil\frac{3 s}{4}\right\rceil-1$ & $t_{\max }=\left\lfloor\frac{3 s}{4}\right\rfloor-1$ & \\
\hline
\end{tabular}

In the following discussion, we use a loose summary of the above table by assuming that $f_{t}(n, s)$ is maximized at $t \sim(3 s / 4)-1$ for large $n$. The reader can refer back to 
the above table for precision.

Recall from an earlier discussion that $M(j, k ; d) \leq f_{d-k-1}(d+1, j)$. The above argument shows that $f_{d-k-1}(d+1, j)$ is maximized for $d-k-1 \sim(3 j / 4)-1$ for large $d$,

$$
M(j, k ; d) \leq f_{(3 j / 4)-1}(d+1, j) .
$$

Given a $j<d$, one can find a $k$ such that $M(j, k ; d)$ attains the upper bound in (31) as follows. Consider a dual-cyclic $j$-polytope with $d+1$ facets $C^{*}(d+1, j)$. From the proof of Theorem 1 , we see that when $C^{*}(d+1, j)$ is realized as the intersection of a $j$-flat $\mathrm{J}$ with $\Delta^{d}$, the $((3 j / 4)-1)$-faces of $C^{*}(d+1, j)$ are realized as the intersections of $\mathrm{J}$ with the $(d-(j / 4)-1)$-faces of $\Delta^{d}$. Setting $k=d-(j / 4)-1$, we see that $J$ intersects $f_{(3 j / 4)-1}(d+1, j) k$-faces of $\Delta^{d}$, which proves the tightness of the bound in (31).

The requirement that $k+j \geq d$ is crucial in the proof of Theorem 3 . When $k+j<d$, if $K_{1}$ and $K_{2}$ are two $k$-faces of $\Delta^{d}$ intersected by a $j$-flat J, then observe that although $J \cap K_{1}$ and $J \cap K_{2}$ are not in general $(k+j-d)$-faces of the $j$-polytope $P=J \cap \Delta^{d}$ (see the discussion following Lemma 1), they are incomparable faces of $\mathrm{P}$ (recall that two faces of a polytope are said to be incomparable if neither is contained in the other). Hence, we conclude that $M(j, k ; d)$ is bounded above by the size of the largest set of incomparable faces in a $j$-polytope with at most $d+1$ facets. In the lattice terminology, a set of incomparable elements in a lattice is called an antichain. Thus, stated differently, $M(j, k ; d)$ is bounded by the size of the largest antichain in the face lattice of a $j$-polytope with at most $d+1$ facets. If $Q$ is an $n$-polytope with $f$-vector $f(Q)=\left(f_{0}, \ldots, f_{n-1}\right)$, then define $f_{\max }(Q)=\max \left\{f_{i}(Q) \mid 0 \leq i \leq n-1\right\}$.

CONJECTURE 1. The size of the largest antichain in the face lattice of an n-polytope with $v$ vertices is at most $f_{\max }(C(v, n))$, where $C(v, n)$ is the cyclic n-polytope with $v$ vertices.

The conjecture, if proved, would imply (together with the asymptotic value of $f_{\max }(C(v, n))$ computed in the proof of Theorem 3$)$ that $M(k, j ; d) \leq f_{(3 j / 4)-1}(C(d+$ $1, j)$ ) for large $d$ even when $k+j<d$.

3. Unresolved problems. In conclusion, we summarize some of the unresolved problems concerning the sections of simplices. We do not believe that the upper bound on $C(j, k ; d)$ is tight. Obtaining tight upper and lower bounds on $C(j, k ; d)$ appear to be very interesting problems geometrically. Apart from the conjecture mentioned above, the problem of obtaining a tight upper bound on $M(j, k ; d)$ which is valid for all $d$ remains open. Finally, while the $f$-vector of a cyclic $d$-polytope is shown to peak around $(3 d / 4)-1$ when the number of vertices is large, location of the maximum in the $f$-vector for small vertex-numbers is not well known although explicit formulae are available for the numbers of faces of every dimension in a cyclic polytope.

ACKNOWLEDGEMENT. This work was supported in part by an NSF Research Initiation Award.

\section{REFERENCES}

[1] A. Bezdek, K. Bezdek, and T. Odor, On a caratheodory-type theorem, preprint(1988). 
[2] K. Bezdek, T. Bisztriczky, and R. Connelly, On hyperplanes and polytopes, Monatsh. Math. 109 (1990), no. 1, 39-48. MR 91e:52015. Zbl 712.52012.

[3] M. R. Emamy K., On the cut-number of the 5-cube, Congr. Numer. 72 (1990), 179-186, Proceedings of the Twentieth Southeastern Conference on Combinatorics, Graph Theory, and Computing (Boca Raton, FL, 1989). MR 91b:05063. Zbl 706.05039.

[4] B. Grünbaum, Convex polytopes, Interscience Publishers John Wiley \& Sons, Inc., New York, 1967. MR 37\#2085. Zbl 163.16603.

[5] A. G. Khovanskii, Hyperplane sections of polyhedra, toric varieties and discrete groups in Lobachevskii space, Funktsional. Anal. i Prilozhen 20 (1986), no. 1, 50-61, 96 (Russian). MR 87k:22015.

[6] P. Kleinschmidt and U. Pachner, Shadow-boundaries and cuts of convex polytopes, Mathematika 27 (1980), no. 1, 58-63. MR 82d:52006. Zbl 428.52004.

[7] P. E. O'Neil, Hyperplane cuts of an n-cube, Discrete Math. 1 (1971;1972), no. 2, 193-195. MR 45 1043. Zbl 218.05003.

[8] G. C. Shephard, Sections and projections of convex polytopes, Mathematika 19 (1972), 144162. MR 47 4142. Zbl 258.52006.

PRABhu: PURdue UnIVERSITY, MGL 1303, West LAFAYETTE, IN 47907, USA 


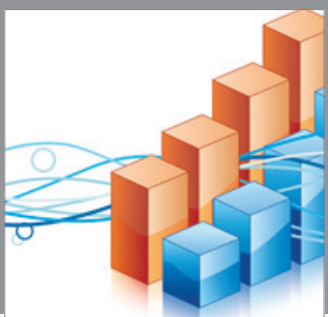

Advances in

Operations Research

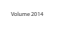

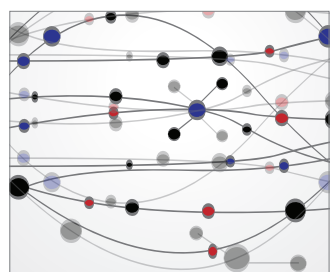

\section{The Scientific} World Journal
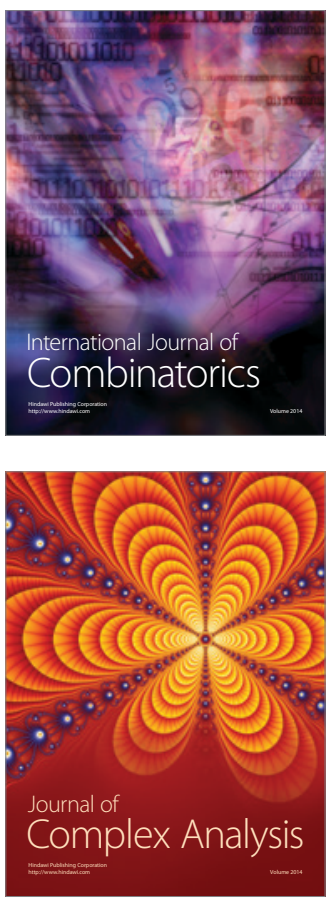

International Journal of

Mathematics and

Mathematical

Sciences
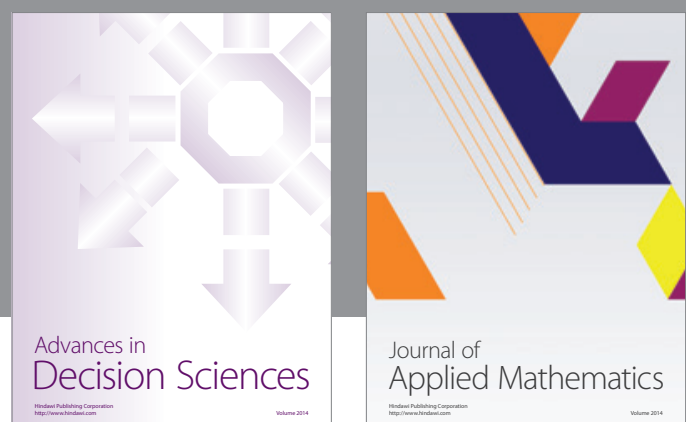

Journal of

Applied Mathematics
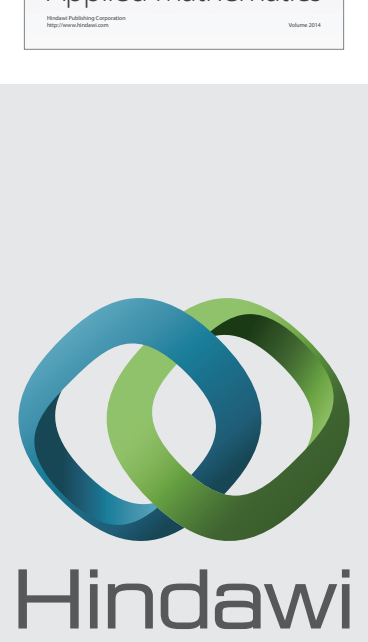

Submit your manuscripts at http://www.hindawi.com
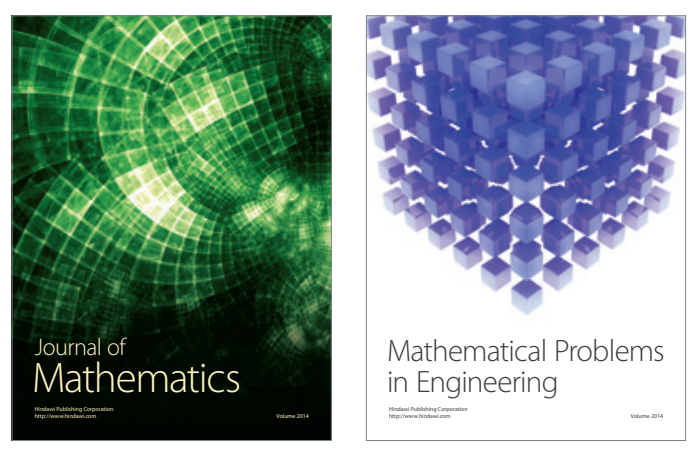

Mathematical Problems in Engineering
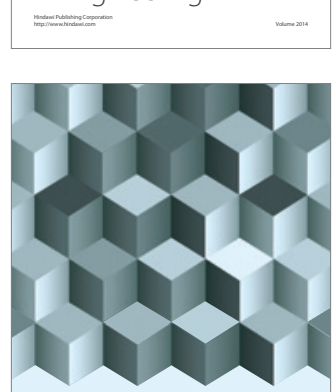

Journal of

Function Spaces
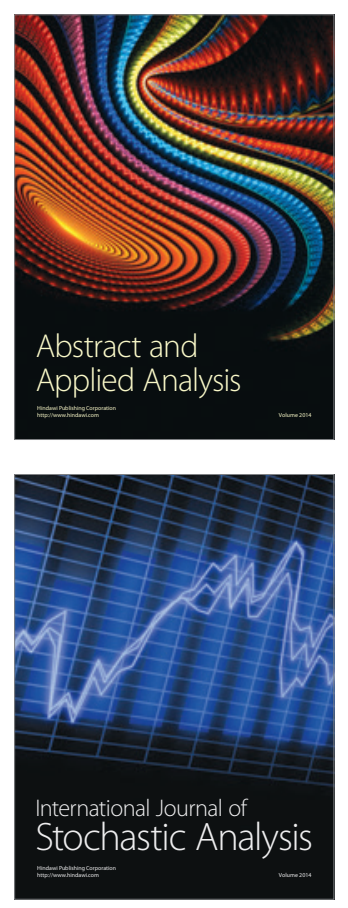

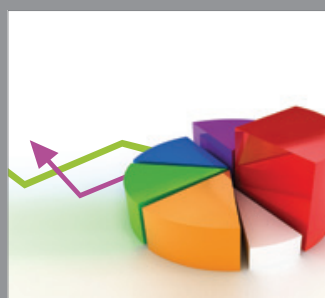

ournal of

Probability and Statistics

Promensencen
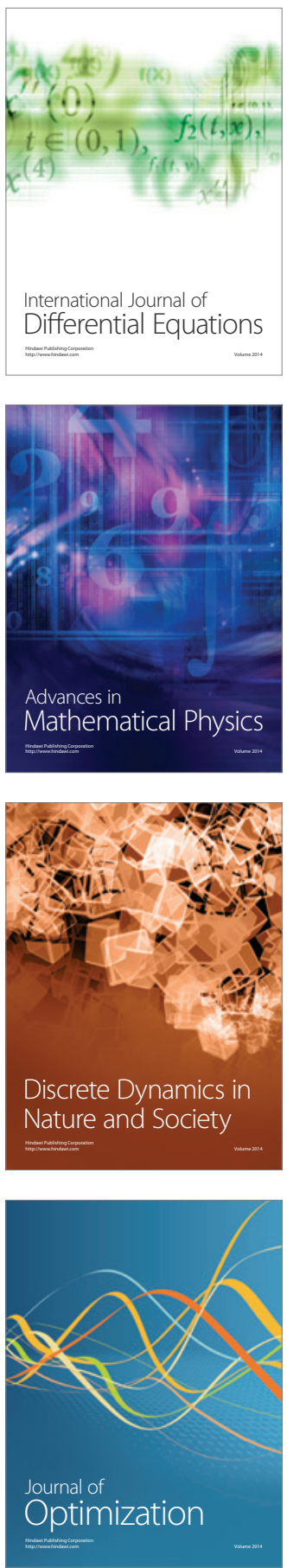